\title{
Nuclear contribution into single-event upset in 3D on-board electronics at moderate energy cosmic proton impact
}

\author{
N. G. Chechenin, T. V. Chuvilskaya and A. A. Shirokova
}

Skobeltsyn Institute of Nuclear Physics, Moscow State University, Moscow, 119991 Russia

\begin{abstract}
In continuation and development of our previous works where nuclear reactions of moderate energy $(10-400 \mathrm{MeV})$ protons with $\mathrm{Si}, \mathrm{Al}$ and $\mathrm{W}$ have been investigated, the results of reactions with $\mathrm{Cu}$ are reported in this paper. $\mathrm{Cu}$ is a most important component in composition of materials in contact pads and pathways of modern and perspective ultra large-scale integration circuitry, especially in 3D topology.
\end{abstract}

It is impossible to imagine a modern spacecraft without electronic integrated schemes with connecting and contact paths and areas, inter-layer conducting connections, and other metallic components. Such interior components normally have a high concentration of $\mathrm{Al}, \mathrm{Fe}, \mathrm{Ni}, \mathrm{Cu}, \mathrm{Mo}, \mathrm{Ta}, \mathrm{W}$, $\mathrm{Pt}, \mathrm{Au}$, and other chemical elements.

The kinematical effect of the energy transfer in a head-on collision from incident proton to a target nucleus is weaker for heavier target nucleus, but the resulting recoil energy can be sufficient to cause a dangerous upset of the electronics in the case of a high energy of incident proton. For example, $1 \mathrm{GeV}$ proton encountering a head-on collision with a gold nucleus loses only about $2 \%$ of its energy but transfers an energy of about $20 \mathrm{MeV}$ to the target gold nucleus. Head-on collisions are quite rare, elastic collisions occur predominantly at nonzero impact parameters, with the result that the kinetic energies of recoil nuclei form a spectrum associated with a specific angular distribution of recoil nuclei $[1,2]$. 
In addition to processes of elastic collisions between protons and target nuclei, it is necessary to consider the contribution of nuclear reactions, which result in the formation of the mass spectrum of recoil nuclei arising as reaction products, which extends from the atomic mass of heavy target nuclei down to helium and hydrogen. Each nuclear-reaction product has its own kinetic-energy spectrum and its own angular distribution. Thus, the picture that arises during the propagation of high-energy cosmic ray particles is quite complicated, so that, in order to estimate quantitatively radiative effects in the onboard electronics, it is necessary to invoke present day nuclear data, as well as modern models and code packages implementing them, which make it possible to compensate for missing nuclear data.

Unfortunately, the presence of heavy elements is disregarded in the majority of the calculations, and this of course impairs the reliability of the respective forecasts. The results of our present calculations make it possible to fill this gap.

Special features of silicon fragmentation induced by high-energy cosmicray protons were explored in previous studies of our group [3-6]. In [5], it was shown that the results of the calculations performed on the basis of the reaction model according to the EMPIRE code are sensitive to the choice of values for the parameters of the optical-model potential. In the present article, the results obtained by calculating for the ${ }^{28} \mathrm{Si}+\mathrm{p},{ }^{27} \mathrm{Al}+$ $\mathrm{p}$ and ${ }^{183} \mathrm{~W}+\mathrm{p}$ nuclear reactions, the cross-sections for elastic and inelastic scattering and the charge, mass, and energy distributions of heavy reaction products according to the TALYS [7] codes are supplemented with the results of the calculations for the same features of the ${ }^{63} \mathrm{Cu}+\mathrm{p}$ reaction.

The idea to create computer codes that would provide a simultaneous description of many nuclear reaction channels as precise as the most detailed description of only one or several reaction channels is not new. Such codes known to date include the GNASH [8], ALICE [9], STAPRE [10] and EMPIRE [11] ones.

The TALYS code [7] is a modern software facility for implementing the modern nuclear-reaction models. It includes the coupled-channel method featuring a number of software procedures. We will now highlight the most important special features of the TALYS code package. First of all, this is a tool for precise calculations of nuclear-reaction mechanisms such as direct, pre-equilibrium and fission ones. The high-precision code makes it possible to construct a reliable description of various reaction mechanisms over a broad region of projectile-particle energies and mass numbers (0.001-200 $\mathrm{MeV}$ and $12<A<239$, respectively). 


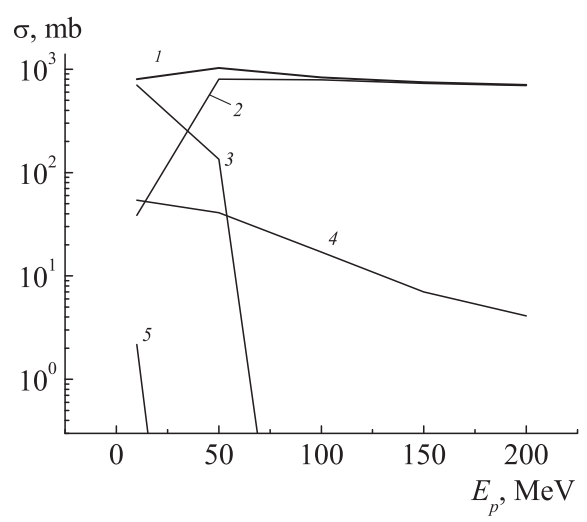

Figure 1: Cross-sections for nuclear processes, $\sigma\left(E_{p}\right)$, in the interaction of protons with a ${ }^{63} \mathrm{Cu}$ nucleus. The curves represent the contributions of (1) inelastic nuclear processes, (2) pre-equilibrium processes, (3) processes leading to compound-nucleus formation, (4) direct nuclear reactions and (5) elastic nuclear processes.

Elastic scattering of protons from a target nucleus with a transfer of a recoil energy to this nucleus is not the most probable mechanism of formation of recoil nuclei. Fig. 1 shows the calculated contributions of the dominant nuclear mechanisms, $\sigma\left(E_{p}\right)$, in the $\mathrm{p}+{ }^{63} \mathrm{Cu}$ reaction at proton energies between 10 and $200 \mathrm{MeV}$. The flux of space protons is strongest in this energy region [12]. The TALYS code package was used in this calculation. Without going into details of the formalism used, we note only that elastic proton scattering from ${ }^{63} \mathrm{Cu}$ nucleus is severely suppressed by competing inelastic nuclear processes (curve 1). The degree of suppression is such that we can disregard elastic scattering. The probability of direct nuclear reactions is somewhat higher (curve 4). They include the direct knockout and pickup of nucleons from the target nucleus without excitation of the residual nucleus. Fig. 1 shows that, in the region of proton energies below $100 \mathrm{MeV}$, a significant contribution comes from reactions involving the production of a compound nucleus (curve 3); at energies below $50 \mathrm{MeV}$, these reactions are dominant. As the proton energy becomes higher, the compound-nucleus mechanism gives way to the competing mechanism of pre-equilibrium processes (curve 2).

It is necessary to consider that the emission of a light particle cascade proceeds within a very short time interval, so that the time of the whole process is less than $10^{-16} \mathrm{~s}$. Within this time, the nucleus involved travels a short distance (smaller than one interatomic spacing in a target material) without causing any shift and ionization of neighboring atoms. We note that 


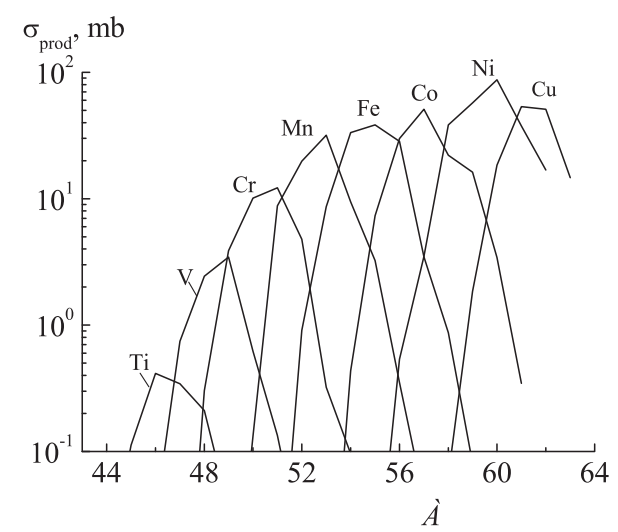

Figure 2: Cross-section for the production of residual nuclei, $\sigma_{\text {prod }}(A)$ (mass spectrum), in the interaction of protons with a ${ }^{63} \mathrm{Cu}$ nucleus at the proton energy of $E_{p}=150 \mathrm{MeV}$.

the production of electron-hole pairs upon medium-atom ionization induced by nuclear fragments is of many orders of magnitude more vigorous than that in the case of primary protons.

The excitation energy of the nucleus involved in the nuclear reaction may be exhausted either by the emission of one particle or by the emission of several particles. As a result, a collision between a proton and a target nucleus leads to the formation of a mass spectrum of residual nuclei. The cross-sections calculated for the production of residual nuclei, $\sigma_{\text {prod }}(A)$, on the basis of modern nuclear-physics models used in the TALYS code package are given in Fig. 2 for isotopes of eight residual nuclei. It can be seen that the product of the reaction ${ }^{63} \mathrm{Cu}(\mathrm{p}, 2 \mathrm{p} 2 \mathrm{n}){ }^{60} \mathrm{Ni}$ is formed with the grate probability. In this reaction, the incident proton leaves part of its energy in the excited nucleus of primary copper. The excitation energy is sufficient for the emission of several protons and neutrons. Isotope production accompanied by the emission of one to ten neutrons has the largest cross-sections ranging from several tens of $\mu \mathrm{b}$ to above $100 \mathrm{mb}$. Ni isotopes of mass number A between 56 and 64 are produced with the largest cross-section. Isotopes of $\mathrm{Cu}$ are produced with a moderately small cross-section below $50 \mathrm{mb}$ in (p,pxn) reaction i.e. via neutron emission and without additional proton emission. As for Co isotopes they are produced in the reaction (p,3pxn) with emission of tree protons. Isotopes of $\mathrm{Fe}$, which is the element that precedes Co in the periodic table of elements, are produced with a rather large cross-section of $40 \mathrm{mb}$ or more in the reaction involving the emission of four protons and 


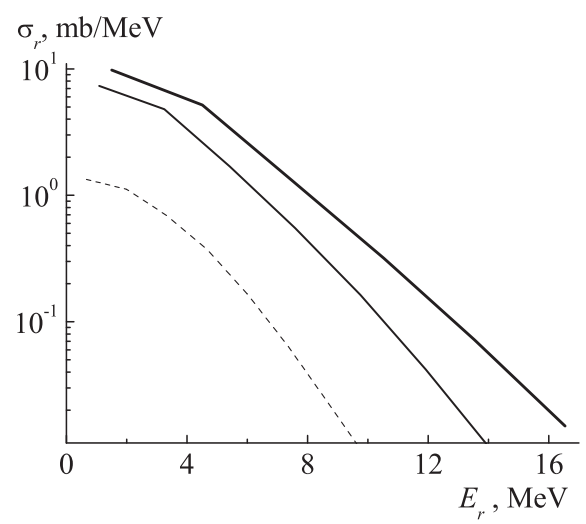

Figure 3: Recoil-energy spectra $\sigma_{r}\left(E_{r}\right)$ of the isotope ${ }^{53} \mathrm{Mn}$ produced in the ${ }^{63} \mathrm{Cu}(\mathrm{p}$, $5 \mathrm{p} 6 \mathrm{n})^{53} \mathrm{Mn}$ reaction versus the product energy at the proton-energy values of $E_{p}=$ 100 (dashed curves), 150 (thin solid curves), and $240 \mathrm{MeV}$ (thick solid curves).

several neutrons. The lower isotope Z-value, the larger number of protons is to be emitted and, hence, the lower cross-section, because of lower penetrability of the Coulomb barrier with de-excitation of the emitting nucleus. The neutron emission becomes dominating in the pre-equilibrium processes. Thus, $\mathrm{V}$ and Ti isotopes are produced with sharply smaller cross-sections.

The kinetic energy distribution of residual nuclei is also of great importance for possible radiation effects in the onboard spacecraft electronics. In Fig. 3 the energy spectra $\sigma_{r}\left(E_{r}\right)$ are shown of ${ }^{53} \mathrm{Mn}$ residual nucleus produced in the $\mathrm{p}+{ }^{63} \mathrm{Cu}$ nuclear reaction for the incident proton energy $E_{p}=100$ (dashed curve), 150 (thin solid curve), and $240 \mathrm{MeV}$ (thick solid curve). This figure shows that the region of kinetic energies of residual nucleus depends both on the incident proton energy and on the mass of the target nucleus. The maximum kinetic energy is observed for the residual nucleus ${ }^{53} \mathrm{Mn}$ at $E_{p}=240 \mathrm{MeV}$. Our calculations have revealed that the recoil energy of residual nuclei may be as high as 1 to $3 \mathrm{MeV}$, depending on the projectile-proton energy.

The results obtained in the present work and outlined partially in this paper seem to be of significance in the estimates of reliability of spacecraft apparatus. 


\section{References}

[1] Pickel J. C., IEEE Trans. Nucl. Sci., 43 (1996) 483.

[2] Chumakov A. I., Effect of space radiation on integral circuitry (in Russian), (Radio I Svyaz, Moscow, 2004).

[3] Chuvilskaya T. V., Shirokova A. A., Kadmenskii A. G., Chechenin N. G., Phys. Atom. Nucl., 71 (2008) 1293.

[4] Chechenin N. G., Chuvilskaya T. V., Shirokova A. A., Kadmenskii A. G., Phys. Atom. Nucl., 72 (2009) 182.

[5] Chechenin N. G., Chuvilskaya T. V., Shirokova A. A., Kadmenskii A. G., Bull. Russ. Acad. Sci. Phys., 74 (2010) 1595.

[6] Chechenin N.G., Chuvilskaya T. V., Shirokova A. A., Kadmenskii A. G., Phys. Atom. Nucl., 78 (2015) 159.

[7] Koning A. J., Hilaire S. et al., TALYS-1.2: A nuclear reaction program (User manual, Netherlands, NRG, 2009).

[8] Young P. G., Arthur E. D. and Chadwick M. B., in Proceedings of the Workshop on Computation and Analysis of Nuclear Data Relevant to Nuclear Energy and Safety, edited by Mehta M. K. and Schmidt J. J., Trieste, Italy, 1993 p. 662.

[9] Blann M., in Proceedings of the Workshop on Computation and Analysis of Nuclear Data Relevant to Nuclear Energy and Safety, edited by Mehta M. K. and Schmidt J. J., Trieste, Italy, 1993 p. 622.

[10] Uhl M. and Strohmaier B., IRK report No. 76/01 Vienna, 1976.

[11] Herman M., in Proceedings of the Workshop on Nuclear Reaction Data and Nuclear Reactors: Physics, Design and Safety, edited by Paver N., Herman M. and Gandini A., Triest, Italy, 2001 p. 137.

[12] Chechenin N. G., Kadmenskii A. G., Motavekh X. A., Panasyuk M. I., Journal of Surface Investigation. X-ray, Synchrotron and Neutron Techniques, 4 (2012) 17. 